\title{
Pancreatitis Aguda en el niño
}

\author{
. II.
}

La pancreatitis aguda es considerada una patología rara en los niños. Estudios nacionales y extranjeros coinciden en que su mayor incidencia se encuentra entre los 30 a 60 años de edad. (4-6).

Revisiones mundiales reportan en total 80 casos de pancreatitis aguda en menores de 16 años. (6).

Richardson en 1895 publica el primer caso de pseudcquiste pancreático en un niño de 13 meses. (2)

Novis en 1923, y Galue en 1924, publican las primeras observaciones de pancreatitis por migracion de ascaris. (2)

Dobbs en 1935 cita 14 casos (2); Adams en 1939. Duncan en 1948, Hargh en 1956. hacen referencia a casos de pancreatitis agu(a. (4). Stein en 1963 reporta en 5 años, 11 casos por infestación parasitaria. (4). Fonkalsrud, en 1968, vublica el estudio de 12 casos con pancreatitis aguda y crónica. en Los Angeles, debida a tratamiento previo con esteroides. (4)

En Chile fueron publicados en 1969, por Aldunate G. y colaboradores: tres casos de lesiones traumáticas del páncreas que están incluidos en la presente revisión y 6 casos por Ossandón y colaboradores en 1974. (5).

No logramos ubicar otras publicaciones nacionales de pancreatitis en la infancia.

*Servicio de Cirtigia. Hospital de Niños Roberto del Río.

\section{MATERIAL CLINICO}

En nutestra revisión encontramos 18 casos rotulados como pancreatitis aguda, de los cuales descartamos 2 fichas por no contar con los exámenes de laboratorio o la comprobación diagnóstica apropiada.

Así presentamos 16 casos de Pancreatitis Agtıda en la Infancia, durante los años 1966 a 1975, atendidos en el Servicio de Cirugía del Hospital Roberto del Río, que fueron comprobados por el laboratorio o por el hallazgo de la lesión durante la laparotomía.

\section{INCIDENCIA: EDAD Y SEXO}

\section{Edad}

Metios 5 años

6 aกีวง-10 aก̃os

1 caso

11 años- 15 aก̃os

5 casos 10 casos

Sexo

Masculino

6 casos

Femenino

10 casos

Notamos que la edad de mayor frecuencia fue entre los 11 y los 15 años (10 casos), y que en el sexo predominó el sexo femenino, 10 casos, sobre 6 del masculino.

En estos dos parámetros: edad y sexo, coincidimos con otros trabajos realizados en el extranjero. (4)

También es de hacer notar que nuestra incidencia es relativamente alta, si la compa- 
ramos con todos Jos casos publicados en la literatura mundial, hasta la fecha (80 casos en niños). (6) Presentamos 16 casos en 10 años en un solo hospital.

\section{ETIOLOGIA}

\begin{tabular}{ll}
\hline Uxlcana & 5 casos \\
Traumática & 4 casos \\
Asociada a colecistopatías & 3 casos \\
Sepsis & 1 caso \\
Desconocida & 3 casos
\end{tabular}

Vemos que la mayoría está representada por las pancreatitis agudas de origen urleano. Tenemos 5 casos que se presentaton entre 4 y 8 dias después de haber presentado parotiditis aguda. Le siguen en frecuencia las pancreatitis agudas de origen traumático: 4 casos, en dos de los cuales se hizo el diagnóstico en una segunda intervención. En la primera intervención se realizó esplecnectomía, debido a estallido dej bazo, posteriormente se siguió el tratamiento babitual, presentándose en los días siguientes agravamiento progresivo con shock y fiebre, obligando a reintervenir, encontrando en el primero de los cascs una fisura de $1 \mathrm{~cm}$. de profundidad en la parte media del páncreas, líquido citrino y gran edema peritoneal: líquido peritoneal con más de $1.000 \mathrm{uW} / 100 \mathrm{ml}$. de amilasa.

En el segundo caso se encontró en la reintervención una lesión traumática de cola y cưrpo del páncreas que obligó a una pancrcatectomía parcial.

Cabe hacer la recomendación de la cuidadosa exploración del páncreas en contusiones abdominales de cierta importancia, en las cuales otros órganos como bazo e hígado han sido lesionados.

Siguiendo con la etiología nos encontramos con otro parámetro de gran inportancia en nuestro medio a diferencia del extraniero: son las pancreatitis agudas asociadas a colecistopatías: colecistitis y colelitiasis. Paredes y Colaboradores (Hospital Arriarán), analizan 150 casos de colecistitis con colelitiasis (7) y en una revisión hecha en nuestro Servicio por el Dr. Jaime Cerda en los últinos 10 años, él presenta 231 casos de colecistitis, de los cuales el $80 \%$ eran litiásicas. (3)

Si comparamos estos estudios con los del extranjero, vemos una gran incidencia de colecistopatías en Chile, lo que podría explicar también la relativa alta incidencia de pancreafitis de los niños en nuestro medio.

Tres pacientes presentaron pancreatitis aguda, sin que fuera posible determinar su etiología; tiso ingresó con la impresión diagnóstica de abdomen agudo y ee encontró la pancreatitis aguda en la laparotomía; otro se ingresó como peritonitis apendicular. Se hicieron los exámenes y el diagnóstico, sin que se llegara a operar. Un tetcer caso ingresó con el diagnóstico de pancreatítis aguda. Se realizaron los exámenes y se comprobó el diagnóstico.

\section{CUADRO CLINICO}

$\begin{array}{lr}\text { Dolor abojominal } & 16 \text { casos } \\ \text { Vómitos } & 13 \text { casos } \\ \text { Fiebre } & 7 \text { casos } \\ \text { Melecrismo } & 6 \text { casos } \\ \text { Mayo-Robson } & 3 \text { casos } \\ \text { Sepsis } & 1 \text { caso } \\ \text { Shock } & 2 \text { casos }\end{array}$

Analizamos ahora las características clínicas de nuestros 16 casos.

Con todos los autores coincidimos en que las caractetísticas clínicas de la pancreatitis aguda son: dolor abdominal, vónitos y metcorismo. $(2,4,6,8)$

\section{EXAMENES DE LABORATORIO}

\begin{tabular}{ll}
\hline Amilasemia & $128 \mathrm{uW} / 100 \mathrm{~m}]-1120 \mathrm{uW} / 100 \mathrm{ml}$ \\
Amilasuria & $257 \mathrm{uW} / 100 \mathrm{ml}-1700 \mathrm{wW} / 100 \mathrm{ml}$ \\
Amilasa & $+1000 \mathrm{uW} / 100 \mathrm{ml}$ \\
Liq. Perit. & +
\end{tabular}

Los cxâmenes de laboratorio nos dieron lo siguiente:

amilasemia (realizada en 12 de los casos) fluctuó entre 120 uW y 1120 uW/100 ml.

amilasuria (rcalizada en 11 de los casos) fíuctuó entre $257 \mathrm{uW}$ y $1700 \mathrm{uW} / 100 \mathrm{ml}$. 
amilasa en líquido petitoneal (realizada en 2 de los 4 casos de origen traumático) fue superior a las $1000 \mathrm{uW} / 100 \mathrm{ml}$.

Se realizaron otros exámenes (calcemia, hemograma, etc.), pero no habiendo sido efectuados en la mayoría de los pacientes, no merccen ser detallados.

\section{DIAGNOSTICO DE INGRESO}

\begin{tabular}{ll}
\hline Pancrcatitis urleana & 5 casos \\
Colecistitis ag. + pancreatitis & 2 casos \\
Puncreatitis de origen inespecífico & 1 caso \\
Perilonitis & 1 caso \\
Colecistitis aguda & 1 caso \\
Contusion abdominal & 4 casos \\
Sepsis, apenclicitis & 1 caso \\
Abdomen agudo & 1 caso
\end{tabular}

En 8 de los 16 casos el diagnóstico fue hecho al ingreso del paciente. Estos fueton:

p a. de origen urleano

5 casos

p. á. asociada a colecistopatías

2 casos

p. a. de etiología no precisada

1 caso

\section{DIAGNOSTICO HECHO POR}

\begin{tabular}{ll}
\hline Clínica y laboratorio & 9 casos \\
C.aparotonja & 7 casos \\
\hline
\end{tabular}

Siete casos fueron diagnosticados por la laparotomia; el diagnóstico preoperatorio fue:

contusión abdominal

colecistitis aguda

sepsis-apendicitis

abdomen agudo

Otro caso ingresó bajo la sospecha de una Peritonitis Apendicular, pero la observación clínica y los exámenes de laboratorio confirmaron la pancreatitis y no se cperó.

\section{TRATAMIENTO}

Siete de los 16 casos fueron tratados con medidas conservadoras dirigidas a:

1. mantener en reposo el tubo digestivo, mediante régimen cero, SNG-aspiración, antiespasmódicos y vagolíticos;

2. mantener el equilibrio hidro-salino;

3. antibióticos de amplio espectro en forma profiláctica;
4. antienzimos (Trasylol) en muy pocos pacientes, por lo cual no merece comentarios.

Los 9 pacientes restantes fueron operados y tratados médicamente con posterioridad.

En los pacientes con PA de origen traumático se realizó evacuación de hematomas, drenaje aspirativo y sutura. En un caso pancreatectomia parcial.

En los casos de PA asociada a colecistopatía se realizó colecistectomía en dos casos y colecistectomía más coledocotomía en un caso.

La evolución inmediata fue satisfactoria; no hubo muertes y en los controles los pacientes han sido considerados como curados.

\section{RESUMEN}

Se analizan 16 casos de Pancreatitis Agucia en la Infancia, en el Servicio de Cirugía Infantil del Hospital Roberto del Río, durante los años 1966-1975, comprobados por clínica, laboratorio $\mathrm{y} / \mathrm{o}$ Iaparotomía.

Se estudia su distribución por edad y sexo, etiología, sintomatología, más frecuente y resultados.

\section{SUMMARY}

16 cases of acute pancreatitis in children are reported, attended at the Pediatric Surgery Service of the Roberto del Rio Hospital since 1966 to 1975 , confirmed with sintomatic picture, labotatory and laparotomy.

We studied its distribution by age and sex, etiology, common sintomatology and the results.

\section{REFERENCIAS}

t. Aldunate $G$, y Colaboradores: Rev, Chil. Pediatr. 40: 1010, 1969.

2. Bienayme, I: Ann. Chir, Inf. 14: 5, 1973.

3. Cerda y Cols.: Colecistitis Aguda en Niños. (En prensa) en Rev. Chil. Pediat.

4 Moosa. A.: Progr. Pediatr. Surg. 4: $111,1972$.

5. Ossandon, F, y Cols.: Rev, Chil. Pediatr. 45:446, 1974.

6. Parada, M.: Arch Soc, Cir, Chile. 19: 23, 1967.

7 Poredes, G. y Cols.: Rev. Chil. Pediatr. 41: 1053, 1970.

8 Salgado, D.: Arch. Soc. Cir. Chile. 19: 51, 1967. 